\title{
A METHOD OF SCALING UP EQUIPMENT FROM THE VIEWPOINT OF ENERGY SPECTRUM FUNCTION
}

\author{
KOHEI OGAWA, CHIAKI KURODA AND SHIRO YOSHIKAWA \\ Tokyo Institute of Technology, Tokyo 152
}

Key Words: Fluid Mechanics, Energy Spectrum, Information Entropy, Eddy Group, Scale-Up, Pipe Flow

How to scale up equipment is a great problem in the chemical industry. Though many methods of scale up have been proposed, none has been established. The main reason is the difficulty in realizing the same mechanism of the turbulent flow field in large-scale equipment as that in the model. Considering that the energy spectrum function (ESF) is one of representative factors which show the mechanism of the turbulent flow field, one of the best ways for scale up is to make the same ESF form in the large-scale equipment as that in the model. The purposes of this paper are to discuss the effects of size of equipment on ESF based on the proposed ${ }^{6)}$ expression of ESF, and to show the care that must be taken in the scale up of equipment by considering developed turbulent pipe flow as an example.

\section{Relationship between Energy Spectrum Function Based on Information Entropy and Pipe Inner Diameter}

An expression of ESF which can apply to wide wavenumber ranges was proposed by using an information entropy in previous papers. ${ }^{5,6)}$ Assuming that a turbulent flow field consists of $m$ eddy-groups and eddy-group $i$ has the average wavenumber $K_{i}$ and takes an ESF form which has the maximum amount of information entropy, the following expression of one-dimensional ESF was proposed.

$$
\frac{E K_{1}}{u^{2}}=\sum_{i=1}^{m} P_{i} \frac{K_{1}}{K_{i}} \exp \left(-\frac{k}{K_{i}}\right)
$$

Additionally, not to speak of the pipe flow fields, many practical ESF in various flow fields were examined and it was made clear that the expression in Eq. (1) can apply to wide wavenumber ranges when the following relations concerning the average wavenumber and the weight factor are satisfied between eddygroup $i-1$ and eddy-group $i$.

$$
\frac{K_{i}}{K_{i-1}}=3, \quad \frac{P_{i}}{P_{i-1}}=\frac{1}{2} \quad(i \geq 2)
$$

Some practical examples of ESF for the axial

\footnotetext{
Received December 3, 1985. Correspondence concerning this article should be
} addressed to $\mathrm{K}$. Ogawa. velocity component in the pipe flow chosen as the subject of study in this paper are shown in Fig. 1. This figure was obtained by moving adequately the curves of ESF for various pipe inner diameters on fulllogarithmic graph paper. The authors' data were some those measured at six radial positions by using an electrode reaction method ${ }^{2}$ in the region of $7500 \leq R e \leq 30,000$. On the other hand, Lawn presented ${ }^{4)}$ ESF data from which it is possible to suppose, when experimental error is considered, that the curve of ESF scarcely depends on the bulk Reynolds number and that the data at any radial position are on one unique curve of ESF. These suppositions were confirmed by making use of the authors' data. Adding the information about ESF described above to this figure, it can be said that the number of eddy-groups $m$ is nearly fixed for each pipe inner diameter and the value of $m$ has a tendency to become large as the pipe inner diameter increases.

From the result that Eq. (2) is valid for pipe flow it can be supposed that turbulent flow field in a pipe is a kind of non-linear vibration system in which subharmonic velocity fluctuations of $1 / 3$ the frequency of the basic velocity fluctuations arises with twice the energy of the basic velocity fluctuations, and that such growth of subharmonic velocity fluctuations occurs one after another. If this supposition is adequate, it is possible to state a reason for the difficulty of scale up. That is, new, larger subharmonic velocity fluctuations should appear if the scale up ratio is larger than 3 . Additionally, it may be possible in some cases that a new larger eddy-group appears even if the scale up ratio is smaller than 3 , and that it. depends on the relationship between the average size of the largest eddy-group and the pipe inner diameter. To make clear whether a new, larger eddy-group appears or not when a pipe is scaled up, the relationship described above must be discussed.

It is possible to estimate the average wavenumber of the smallest eddy-group $K_{m}$, the reciprocal of which represents the average size of the smallest eddy group, by making use of Eqs. (1) and (2) and experimental results. The values for the water and air flows can be 


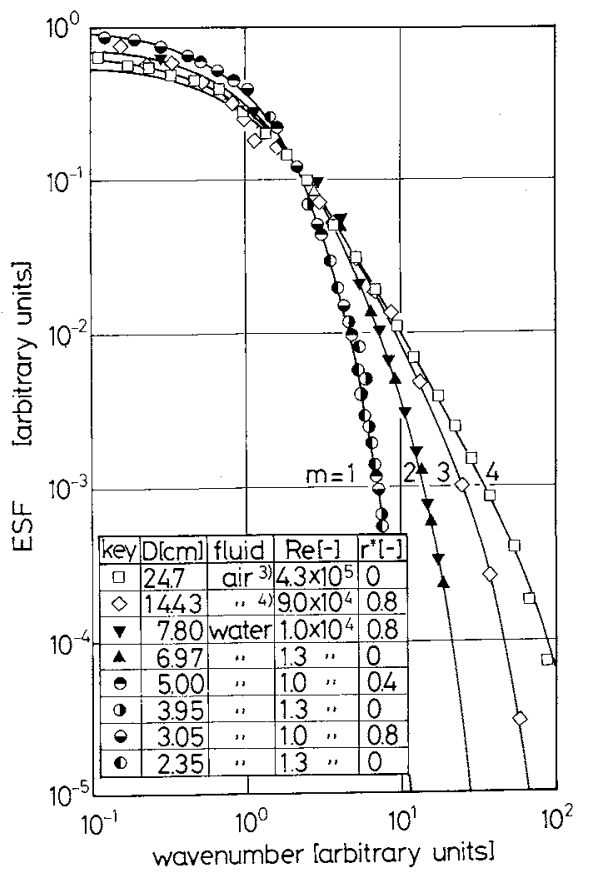

Fig. 1. ESF for axial velocity component in pipe flow.

determined as follows.

$$
\begin{gathered}
\text { water: } K_{m}=1.40 \mathrm{~cm}^{-1} \\
\text { air: } K_{m}=5.70 \mathrm{~cm}^{-1}
\end{gathered}
$$

These values are smaller than the wavenumber ranges in which the main energy dissipation occurs. ${ }^{1)}$ It is suitable to suppose the following relational equations between the values of the pipe inner diameter $D$ and the representative average size of the largest eddygroup $\alpha 3^{m} / K_{m}$.

$$
\alpha \frac{3^{m-1}}{K_{m}}<D \leq \alpha \frac{3^{m}}{K_{m}}
$$

where $\alpha$ is the proportional constant that relates the reciprocal of the average wavenumber $K_{1}$ and the pipe inner diameter $D$. By using the $K_{m}$ values determined above, the range of values of constant $\alpha$ that satisfies all experimental data for each fluid can be determined. Almost all the ranges for both fluids overlapped each other in the following ranges:

$$
2.89 \leq \alpha \leq 3.60
$$

and it can be said that the value of the constant does not depend on the fluids. The average value of the range is determined as $\alpha_{a}=3.08$. If this value of $\alpha_{a}$ is used, the relationship between the number of eddygroups $m$ and the pipe inner diameter $D$ is obtained as shown in Table 1. In Fig. 2, the relationship between $K_{m}$ or $K_{1}$ and the pipe inner diameter is shown.

Therefore, it is possible to estimate roughly the critical scale up ratio at which a new larger eddygroup appears in a large-scale pipe by using this table, and it may be easy to estimate the form of ESF of
Table 1. Relationship between eddy-group number and pipe inner diameter

\begin{tabular}{ccc}
\hline$m$ & Water & Air \\
\hline 1 & $0<D \leq 6.6 \mathrm{~cm}$ & $0<D \leq 1.62 \mathrm{~cm}$ \\
2 & $6.6<D \leq 19.8$ & $1.62<D \leq 4.86$ \\
3 & $19.8<D \leq 59.4$ & $4.86<D \leq 14.6$ \\
4 & $59.4<D \leq 178$ & $14.6<D \leq 43.8$ \\
5 & $178<D \leq 535$ & $43.8<D \leq 131$ \\
6 & $535<D \leq 1604$ & $131 \quad<D \leq 394$ \\
\hline
\end{tabular}

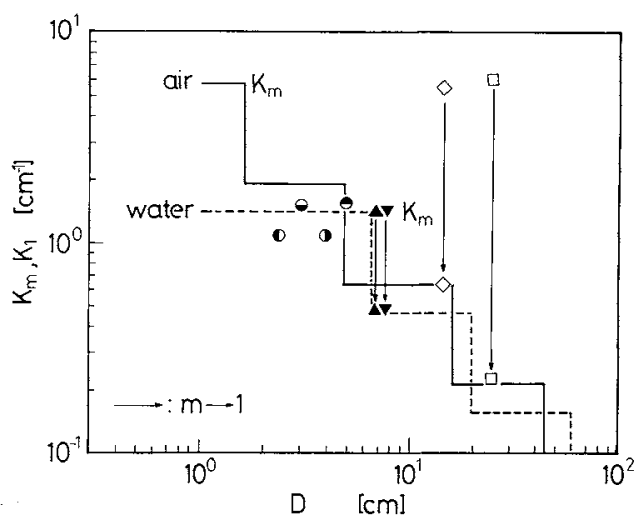

Fig. 2. Relationship between $K_{m}$ or $K_{1}$ and pipe inner diameter. $(\rightarrow$ denotes the change in the average wavenumber of the largest eddy-group for each experiment.).

fluid flow in a pipe with arbitrary inner diameter by using Eqs. (1) and (2) and Table 1.

\section{Steps to Scale-up}

According to the way of thinking about ESF described above, the following is the best method for scale up of pipes in which water or air flows with developed turbulence.

1. The cross-sectional average velocity in the large-scale pipe is set to be the same as that in the model. (It was confirmed experimentally by this work that the turbulent energy is in proportion to the square of the cross-sectional average velocity.)

2. ESF in the model is measured and the value of $m$ is determined by using Eqs. (1) and (2) or Fig. 1 or Table 1 .

3. The value of $m$ in the large-scale pipe is estimated by using the relationship between the pipe inner-diameter and the value of $m$ by using Table 1 .

4. When the value of $m$ in the large-scale pipe is the same as that of the model, the scale up is accomplished. When the value of $m$ in the large-scale pipe is larger than that in the model, the scale up ratio should be decreased until the value of $m$ in the largescale pipe becomes the same as that in the model. When it is impossible to decrease the scale up ratio in the case described above, the cross-sectional average velocity in the large-scale pipe is arranged so that the turbulent energy of the limited wavenumbers which are considered to play the significant roles for the 
intended use of the pipe becomes the same as that in the model.

It can be said that this stepwise approach to scale up might be applied to the scale up of other equipment as well.

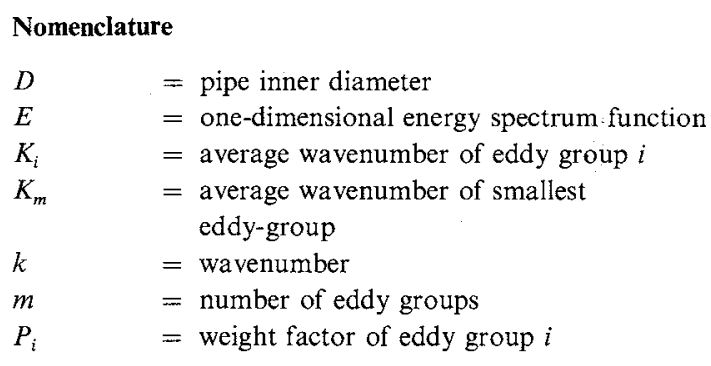

$$
\begin{array}{llr}
R e & =\text { Reynolds number } & {[-]} \\
r^{*} & =\text { dimensionless radius by using } D & {[-]} \\
u & =\text { root-mean-square turbulent velocity } & {[\mathrm{m} / \mathrm{s}]} \\
\alpha & =\text { constant }
\end{array}
$$

\section{Literature Cited}

1) Hinze, J. O.: "Turbulence," 2nd ed., McGraw-Hill, New York (1975).

2) Ito, S. and K. Ogawa: J. Chem. Eng. Japan, 6, 231 (1973).

3) Laufer, J.: NACA TN1174 (1954).

4) Lawn, J.: J. Fluid Mech., 48, 477 (1971).

5) Ogawa, K.: J. Chem. Eng. Japan, 14, 250 (1981).

6) Ogawa, K., C. Kuroda and S. Yoshikawa: J. Chem. Eng. Japan, 18, 544 (1985).

\title{
EFFECT OF OXIDATION CONDITIONS ON Ni CRYSTALLITE SIZE IN PREPARATION OF CATALYSTS FROM RARE EARTH INTERMETALLIC COMPOUNDS
}

\author{
TADASHI SHIROTSUKA, KAORU ONOE AND AYUMU YOKOYAMA \\ Department of Applied Chemistry, Waseda University, Tokyo 160
}

\begin{abstract}
Key Words: Chemical Reaction, Rare Earth Metal, Intermetallic Catalyst, Oxidation, Nickel Crystallite
\end{abstract}
Size, Diffusion Coefficient

Recently, intermetallic compounds containing rare earth metals and transition metals have been found to be active catalysts for CO hydrogenation. ${ }^{1)}$ Work on $\mathrm{LaNi}_{5}{ }^{5)}$ has shown that it decomposed into $\mathrm{Ni}$ and $\mathrm{La}_{2} \mathrm{O}_{3}$, and this decomposed product $\left(\mathrm{Ni} / \mathrm{La}_{2} \mathrm{O}_{3}\right)$ is the actual catalyst. This work suggests that oxidation of intermetallic compounds is useful in producing new catalysts.

The previous study ${ }^{4}$ focused on oxidation treatment of $\mathrm{LaNi}_{5}$ as a new means of forming $\mathrm{Ni}$ catalysts without such disadvantages as the poisoning effect of residual ions, which are often encountered with conventional methods using aqueous solutions.

The present study was undertaken to investigate the effects of oxygen diffusion rate and oxidation temperature on the size of $\mathrm{Ni}$ crystallites obtained from three intermetallic compounds- $\mathrm{CeNi}_{5} . \mathrm{LaNi}_{5}$ and $\mathrm{SmNi}_{5}$. The oxidation temperature was found to influence Ni crystallite size more strongly than differences in the crystal structures of rare earth oxides.

Received February 1, 1986. Correspondence concerning this article should be ad dressed to K. Onoe.

\section{Experimental}

\subsection{Materials}

$\mathrm{CeNi}_{5}, \mathrm{LaNi}_{5}$ and $\mathrm{SmNi}_{5}$ were prepared by induction melting of the metal components in a watercooled copper boat under a purified argon atmosphere. The purity of the metal components was $99.8 \mathrm{wt} \%$ for $\mathrm{Ce}, \mathrm{La}$ and $\mathrm{Sm}(0.2 \mathrm{wt} \%$ of other rare earths), and $99.97 \mathrm{wt} \%$ for $\mathrm{Ni}(0.03 \mathrm{wt} \%$ of $\mathrm{Cu}, \mathrm{Co}$ and $\mathrm{Fe}$ ). The compounds were remelted several times to ensure homogeneity, and structure was verified by $\mathrm{X}$-ray diffraction analysis.

\subsection{Analytical procedure}

The analytical procedure was similar to that previously used, and is described elsewhere. ${ }^{43}$ It involved the use of a thermogravimeter for weight measurement, powder X-ray diffraction for observing the transformation, X-ray diffraction line-broadening for $\mathrm{Ni}$ crystallite size measurement, the BET method for surface area measurement, and emery-grinding for observing the vertical section of oxidized samples. 